\title{
HELICAL BUCKLING OF DRILL-STRINGS
}

\author{
Marcin Kapitaniak ${ }^{1, \star}$, Vahid Vaziri ${ }^{1}$, and Marian Wiercigroch ${ }^{1}$ \\ ${ }^{1}$ Centre for Applied Dynamics Research, School of Engineering, University of Aberdeen, Aberdeen, AB24 \\ 3UE, Scotland, UK
}

\begin{abstract}
This paper presents experimental and numerical investigations of the complex drill-string vibration, with particular attention to the helical buckling of drill-strings and its influence on the system dynamics. The analysis is conducted using a novel experimental drilling rig, capable of exhibiting of all major types of drill-string vibration, including torsional, axial and lateral modes, which utilizes real industrial drill-bits and rock samples. A good qualitative and quantitative agreement between the experiments and numerical model is demonstrated.
\end{abstract}

\section{Introduction}

The drill-strings used for oil and gas extraction, due to their length reaching up to several kilometers, have a very low bending stiffness compared to the torsional one, what makes them impossible to be driven it in a completely straight configuration. Thereby the drill-string will buckle under its own weight and as the rotation from the top is applied, will take a helical shape due to the constraint in a form of the borehole. Due to the multiple contact points between the drill-string and the borehole, as well as the helical shape the equivalent properties of the structure are different than for the straight configuration. This can have a significant influence on the system dynamics, leading for example to well known stick-slip phenomenon at the drill-bit rock interface, which in turn can result in reduced efficiency of the drilling process, increased wear of the equipment and subsequent catastrophic failures [1]. The aim of this paper is to present the experimentally calibrated model, that takes into account helical buckling of drill-strings and provides further capabilities and understanding comparing to other models [2-4].

\section{Aberdeen drilling rig}

In order to gain a deeper understanding of drillstring vibration, a novel experimental drilling rig [2] has been constructed at the University of Aberdeen, which is capable of reproducing stick-slip, whirling and bit-bounce phenomena. In this paper we focus on the torsional buckling of the drill-string and demonstrate that it can be a cause of stick-slip oscillations. In our experimental facility, the drill-string is driven from top by a $3 \mathrm{~kW}$ electric motor which can provide up to $1032 \mathrm{rpm}$. Depending on the chosen configuration either rigid or flexible shafts are used to transmit rotary motion to the Bottom Hole Assembly (BHA) section and ultimately to the drill-bit. The BHA is made of a heavy steel shaft, held in transversal direction using a loose bearing, as shown schematically in Fig. 1 (a). An axial static force or a Weight on Bit (WOB) is realized by placing steel disks on the top of

^e-mail: marcin.kapitaniak@abdn.ac.uk 


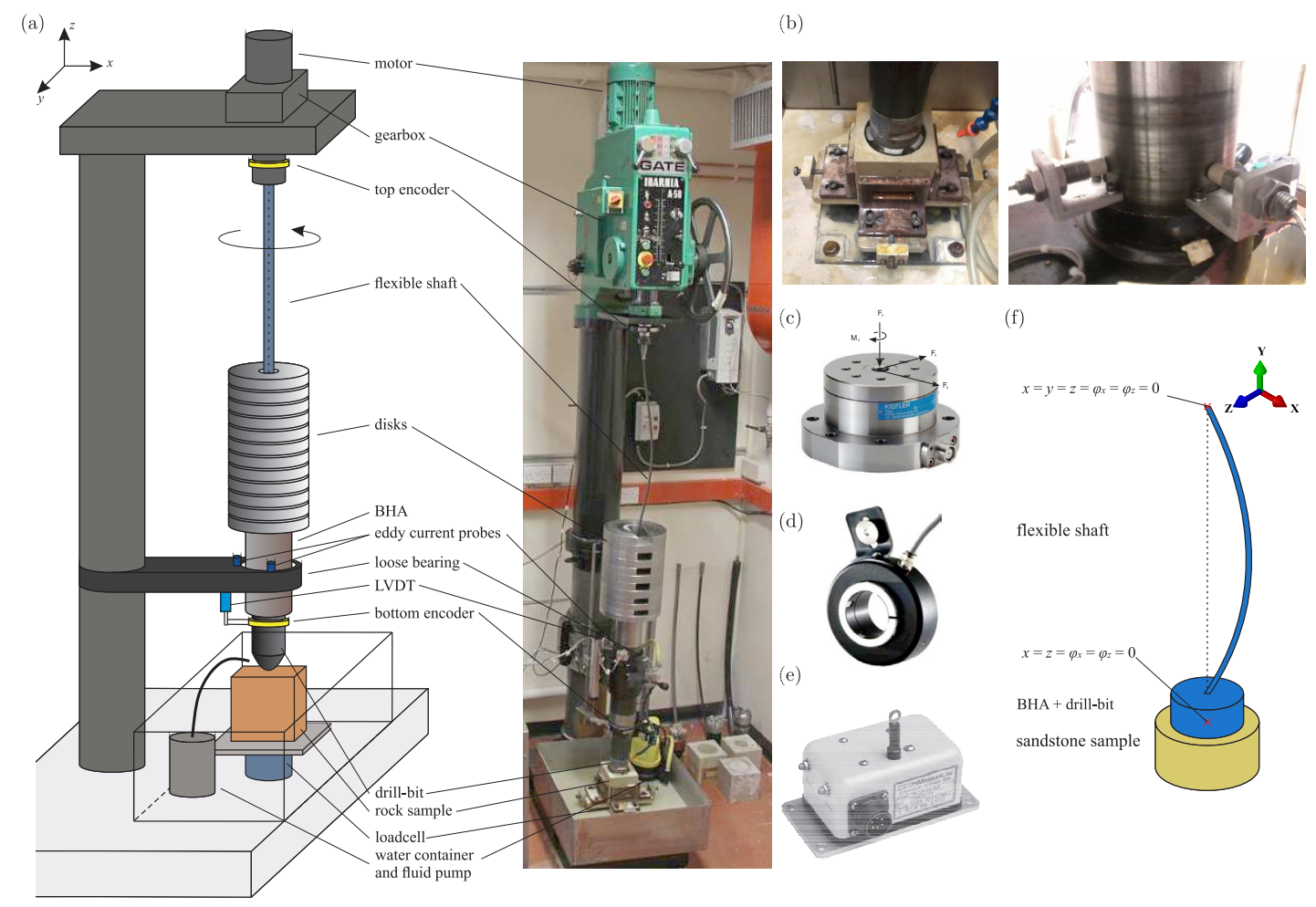

Figure 1. (a) Schematic diagram and photograph of the experimental setup. Main components of the system are: electric motor, flexible or rigid shafts, disks, the BHA, drill-bit and rock samples. (b) Drill-bit rock interface and the BHA with position of the eddy current probes, (c) 4-component load cell (d) top and bottom encoders and (e) LVDT. (f) Configuration of the FE model for pre-buckled configuration, showing all the boundary conditions applied at the top and bottom nodes.

the BHA providing WOB within the range 0.93 to $2.79 \mathrm{kN}$. A variety of different sensors are used in the experimental setup, which allow to conduct detailed measurements of the most important variables of the drilling process. These include two incremental rotary quadrature encoders (Fig. 1 (d)) to measure top and bottom angular velocity, an axial position transducer (Fig. 1 (e)) to observe Rate of Penetration (ROP) and most importantly two eddy current probes to measure the lateral position of the BHA within the range $0-5 \mathrm{~mm}$ (see Fig. 1 (b)). The most advanced sensor in our setup is the four component dynamometer (Kistler 9272), placed directly below the rock sample, which allows to measure the axial force $F_{z}$, reaction torque $T_{z}$, and two forces acting in transversal directions $F_{x}$ and $F_{y}$ as depicted in Figure 1 (c). The ranges of measurement for Torque on Bit (TOB) and WOB are: $\pm 200 \mathrm{Nm}$ and $-5-20 \mathrm{kN}$ respectively. For more details about the experimental rig, please refer to [5-7]. 


\section{Experiments and Modelling}

In our work we perform experimental observations of the stick-slip phenomenon, with the drill-string in the buckled configuration and on this basis calibrate a Finite Element (FE) model of the rig. Note that in the experimental analysis we represent the drill-string section using flexible shaft of very low bending to torsional stiffness ratio. As can be seen in Fig. 1(f), the FE model of the experimental setup consists of two cylinders, one representing the drill-bit and the other the rock sample, as well as the flexible shaft set in the prebuckled configuration, which allows us to observe helical buckling condition. As the flexible shaft exhibits different properties depending on the direction, it is required to describe it as an orthotropic material, that has different properties along 3 principal axes. This means 9 independent material constants are required to form elasticity matrix $\mathbf{D}$ to describe the stress-strain relationship. One of the critical steps in the modelling of drill-string dynamics is the mathematical description of the bit-rock interactions. Quantitatively, these interactions are represented in terms of TOB versus bit velocity curves, which in turn allow us to compute equivalent friction coefficient (slip-rate dependent) that captures the main phenomena observed in our drilling tests, namely, cutting and friction between the drill-bit and the rock sample. In the FE model, we assign following friction coefficient between the disc representing the drillbit and the BHA and the one representing the rock sample: $\mu=\mu_{k}+\left(\mu_{s}-\mu_{k}\right) e^{-d_{c} r \dot{\varphi}_{b}}+\mu_{s t r} r \dot{\varphi} b$, where $\mu_{k}, \mu_{s}, \mu_{s t r}, d_{c}, r$ and $\dot{\varphi}_{b}$ represent the kinetic, static, Stribeck friction coefficient, the decay rate, drill-bit radius and angular velocity respectively. For calibration details of the FE model of the flexible shaft and the drill-bit rock interactions please refer to [6].

An example of experimental time history and phase portrait (marked in red), clearly showing stick-slip behaviour, is shown in top panel in Fig. 2 (c), which is compared with the response from calibrated FE model of the assembly (in green) below. Note that the same top excitation is applied in experiment and the FE model (black curve), which assigns following inertia properties to the disc representing the BHA ( $m=$ $148.579 \mathrm{~kg}$ and $\left.J=0.8416 \mathrm{kgm}^{2}\right)$, elastic properties of the flexible shaft (Young moduli: $E_{1}=$ $E_{2}=3.64 \mathrm{GPa}, E_{3}=1.50 \mathrm{GPa}$, shear moduli: $G_{12}=0.02 \mathrm{GPa}, G_{13}=G_{23}=18.16 \mathrm{GPa}$, Poisson ratios: $\left.v_{12}=v_{13}=v_{23}=0.30\right)$ and equivalent friction coefficients $\left(\mu_{s}=0.1280\right.$, $\left.\mu_{k}=0.0874, \mu_{s t r}=0.0085, d_{c}=2.2047\right)$, that correspond to experimental values, as identified in [6]. A very good qualitative and quantitative agreement is achieved, which is particulary visible when comparing shapes of the flexible shaft during different stages of the stick-slip motion, corresponding to experiment and the FE model (See front and top views shown in Figs 1 (a) and (b) respectively). Note, that there are small differences, as the model predicts slightly smaller amplitude of stick-slip vibrations, while keeping the same period. At the same time, when comparing the phase portraits for both cases, we observe that the sticking interval is also slightly smaller than the one observed in experiment, which might be caused by the simplification of cutting process using an equivalent friction approach.

Additionally, we observe experimentally the variation in WOB and in TOB, which can be directly related to the sticking phases (points AB) visible in angular velocity of the drill-bit, as shown in left hand side panels of Figs 2 (d)-(f). This behaviour is confirmed in the FE model with corresponding time histories shown in the right panels (Figs 2 (d)-(f)), which provides further validation for the model's calibration. As shown in Fig. 2 (e), in both the experimental and numerical results, there is a drop in WOB exactly at a point where sticking phase begins (point A). This suggests an upward force acting on the drill-bit, due to helical deformation of the flexible shaft and elastic energy accumulated. As for the TOB, its variations happen mainly due to the changes of the velocity of the drill-bit and frictional/cutting characteristics of the drill-bit-rock interface. Looking closely on the time history of TOB, we observe that exactly on the onset of sticking phase (point A) a clear sudden increase of TOB is visible, indicat- 

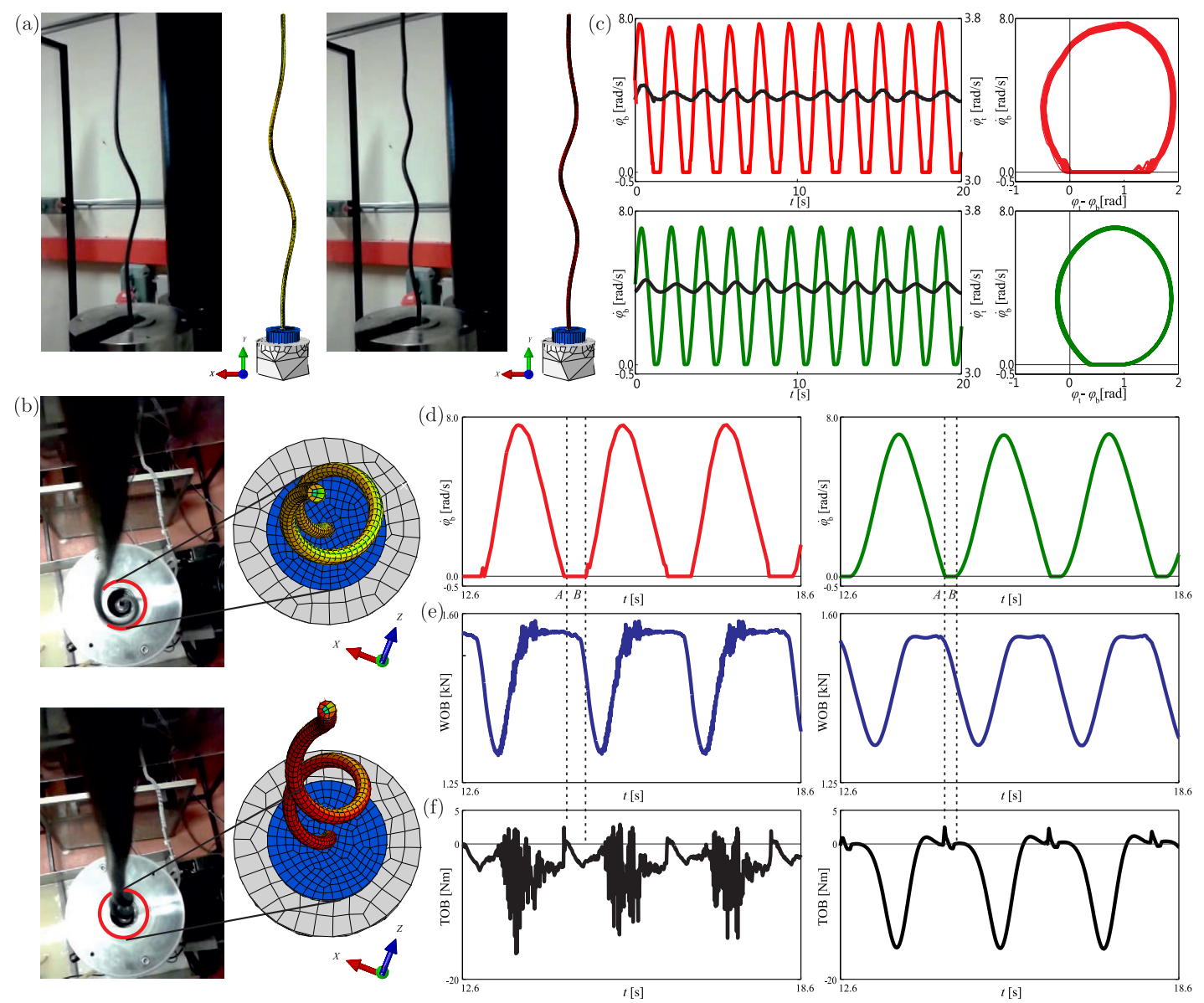

Figure 2. (a)-(b) Comparison of shape of the flexible shaft during stick-slip oscillations for experiment and FE model for prebuckled configuration $\left(d_{d b}=0.0381 \mathrm{~mm}\right)$ and WOB of $1.45 \mathrm{kN}$ (front and top views respectively). (c) Corresponding time histories and phase portraits for experiment (in red) and FE model (in green). (d)-(f) A detailed view into time histories of bit angular velocity $\dot{\varphi_{b}}$, WOB and TOB respectively (Left and right panels depict experimental and numerical responses respectively).

ing reaching a static torque. During the sticking

\section{Conclusions}

In this paper, we focus on the experimental verification of the developed FE model of the drilling rig introduced in $[7,6]$. The drillstring (represented by the flexible shaft in the experiment) is driven in a buckled configuration, which allows us to investigate effects of heli- phase, TOB decreases to the initial value by the time slip phase occurs again (point B).

cal buckling on stick-slip motion. After careful calibration of the FE model, we are able to achieve the qualitative and quantitative agreement between experiments and the model. The developed model is capable of predicting accurately the shape of the flexible shaft during different phases of stick-slip phenomena as well as 
changes in TOB and WOB, as shown in detail in Figs. 2 (a)-(f). In view of positive verification of the developed FE model, we can conclude that the model applicability has been demonstrated, which will allow for further investigation into the complexity of helical buckling phenomenon.

\section{References}

[1] A.M Chevallier, N.P Politis, M.L Payne, and P.D. Spanos, Shock and Vibration Digest, 35(2), 85-103 (2003)

[2] H. Al-Naser and Y.A. Khulief, Finite Elements in Analysis and Design, 41(13), 1270-1288 (2005)
[3] C. Liao, B. Balachandran, M. Karkoub, and Y.L. Abdel-Magid, Journal of Vibration and Acoustics, 133(4), (2011)

[4] E. M. Navarro-López and D. Cortés, Journal of Sound and Vibration, 307, 152-171 (2007)

[5] M. Kapitaniak, V. Vaziri Hamaneh, J. Páez Chávez, and M. Wiercigroch, International Journal of Mechanical Sciences, 101-102, 324-337 (2015)

[6] M. Kapitaniak, PhD thesis, University of Aberdeen, (2015)

[7] M. Kapitaniak, V. Vaziri Hamaneh, J. Páez Chávez, and M. Wiercigroch, Mechanical Systems and Signal Processing, 100, 454-465 (2018) 